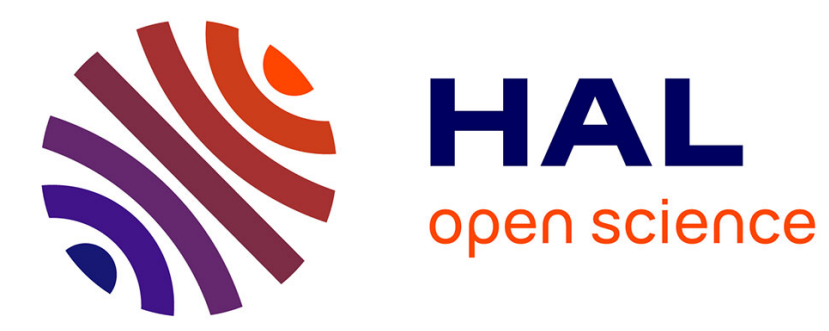

\title{
Splittable Routing Games in Ring Topology with Losses
}

\author{
Sami Dallali, Clara Fontaine, Eitan Altman
}

\section{To cite this version:}

Sami Dallali, Clara Fontaine, Eitan Altman. Splittable Routing Games in Ring Topology with Losses. ASMTA 2021 - 26th International Conference on Analytical \& Stochastic Modelling Techniques \& Applications, Dec 2021, Virtual, Japan. 10.1007/978-3-030-91825-5_18 . hal-03340020

\section{HAL Id: hal-03340020 \\ https://hal.science/hal-03340020}

Submitted on 9 Sep 2021

HAL is a multi-disciplinary open access archive for the deposit and dissemination of scientific research documents, whether they are published or not. The documents may come from teaching and research institutions in France or abroad, or from public or private research centers.
L'archive ouverte pluridisciplinaire HAL, est destinée au dépôt et à la diffusion de documents scientifiques de niveau recherche, publiés ou non, émanant des établissements d'enseignement et de recherche français ou étrangers, des laboratoires publics ou privés. 


\title{
Splittable Routing Games in Ring Topology with Losses
}

\author{
Sami Dallali ${ }^{4}$, Clara Fontaine ${ }^{4,5,6}$, and Eitan Altman ${ }^{1,2,3}$ \\ 1 INRIA Sophia Antipolis - Méditerranée; \\ 2 CERI/LIA, University of Avignon, FR \\ 3 lincs, 23 Ave d'Italie, 75013 Paris \\ 4 CentraleSupélec, CS, Paris Saclay, Gif sur Yvette, FR \\ ${ }^{5}$ Cornell University, Engineering Management and EE, USA \\ ${ }^{6}$ Centre for Quantum Technologies at the National Univ of Singapore, \\ email: sami.dallali@student-cs.fr, cyf3@cornell.edu, Eitan.Altman@inria.fr
}

\begin{abstract}
We consider a splittable atomic game with lossy links on a ring in which the cost that each player $i$ minimizes is their own loss rate of packets. The costs are therefore non-additive (unlike costs based on delays or tolls) and moreover, there is no flow conservation (total flow entering a link is greater than the flow leaving it). We derive a closed-form for the equilibrium, which allows us to obtain insight on the structure of the equilibrium. We also derive the globally optimal solution and obtain conditions for the equilibrium to coincide with the globally optimal solution.
\end{abstract}

Keywords: Routing games · Loss probabilities · Ring topology

\section{Introduction}

We study routing on a ring network in which traffic originates from nodes on the ring and is destined to the center node. Each node has two possible paths: either a direct path from the node to the center node or a two-hop path in which the packet is first relayed to the next node on the ring and then takes the direct link from that next node to the common destination. The traffic originating from a given node is assumed to form an independent Poisson process with some intensity (which we call the demand). Beyond forwarding the traffic that arrives from the previous node, each node has to decide what fraction of its own traffic would be routed on each one of the two possible paths to the destination.

Routing games of this type have been intensively studied both in the road traffic community [13] as well as in the community of telecommunications network [12] under additive costs (such as delays or tolls) and conservation constraints (at each node, the sum of incoming flow equals the sum of outgoing flows). In this paper, we depart from these assumptions by considering loss networks in which losses may occur at all links: there are links with i.i.d. losses 
(relay links) and collision losses (on direct links between source and the common destination node).

Two levels of system modeling are presented here: a flow level in which routing decisions are taken, and a more detailed packet-level modeling that determines the losses and thus the interference between flows from different sources. The decisions of a node concern only the fraction of packets originated in that node which will be routed on each of the two paths available to traffic from that node. Then the actual packets to be transmitted over each one of the paths are selected according to an i.i.d. Bernoulli thinning process. The decisions are thus the Bernoulli thinning parameters.

The ring topology has received much less attention than the parallel-link topology as well as the load-balancing triangular topology introduced by Prof Kameda and his students. Although in practice, the ring topology may seem to be a toy problem, we do encounter ring networks quite often in practice, mostly in runabouts. Ring topologies can also be found in access to communication networks, both in local area networks - see IEEE 802.5 token ring standard and the metropolitan area network FDDI [4].

Related work Previous studies of routing games with circular topology have appeared in citecircle1,burra,chen. $[7,8]$ consider linear costs, and none of these references consider non-conservation of flows. We note also that in [1], there are either bi-directional roads or two rings, one in each of two directions (clockwise and anti-clockwise), and cars have to decide the direction to drive. In [3] other non-additive cost criteria have been introduced in a context of load-balancing games (triangle topology) where their performance measure is related to blocking probabilities. See also [2] and [5] for other related work.

Focusing on symmetric ring networks, our main contribution is to obtain closed-form expressions with the help of Maple. This includes best response functions, derivatives of the costs that are used to compute the best response, and the symmetric equilibrium. We derive the globally optimal solution as well as the equilibrium solution.

\section{The model}

We consider $K$ nodes on a circle, indexed by $0,1, \ldots, K-1$, see Figure 1 .

Each node $k$ is connected to a set $N_{k}$ containing $N$ players. Each player $(n, k) \in N_{k}$ has to ship a strictly positive demand $\phi_{k}$ to a destination $\Delta$ common to all players. Each of the $N k$ players generates packets following an independent Poisson process. The player decides with what probability to send an arrival that it generates over one of two possible paths to $\Delta$; the packet can use a direct transmission link $D(k)$ or an indirect path consisting of first relaying the packet to node $\mathrm{k}+1$ and only then transmit it to $\Delta$ through $D(k+1)$ (note that node indices are modulo $K$.) Let $x_{k}^{n}$ and $\alpha_{k}^{n}$ denote the amount and fraction, respectively, of class $\mathrm{k}$ flow originating from player $(n, k)$ through the direct path, i.e., through link $D(k)$. We call

$$
\alpha=\left(\alpha_{k}^{n}, k=0, \ldots, K-1, n=1, \ldots, N\right)
$$




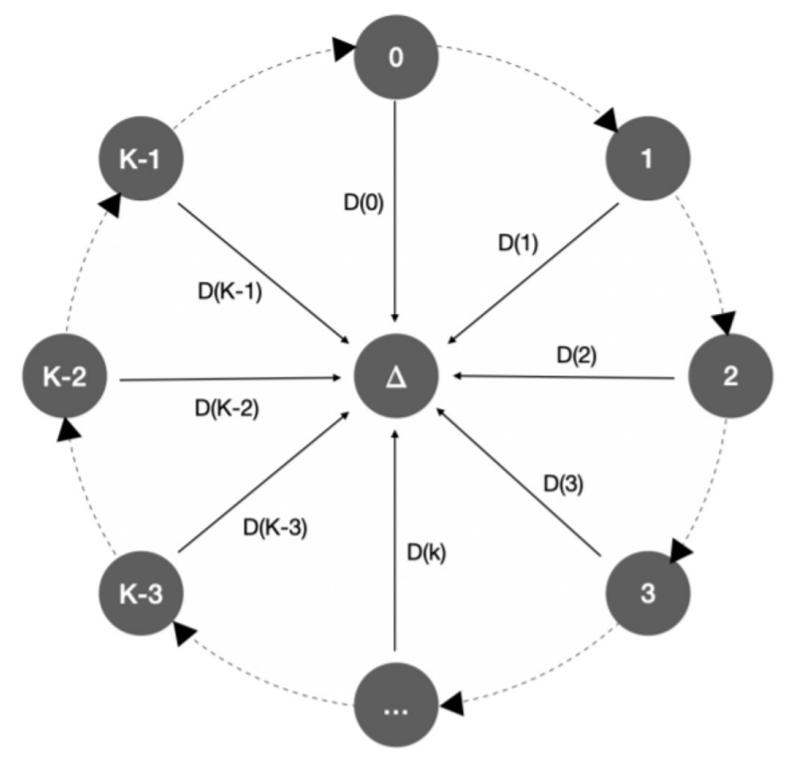

Fig. 1. Ring Network Topologu

the assignment or th action vector. For a given demand vector $\phi=\left(\phi_{k}^{n}, k=\right.$ $0, \ldots, K-1, n=1, \ldots, N)$, the assignment vector $\alpha$ completely specifies the set of flows

$$
\mathbf{x}=\left(x_{k}^{n}, k=0, \ldots, K-1, n=1, \ldots, N\right)=\left(\alpha_{k}^{n} \phi_{k}^{n}, k=0, \ldots, K-1, n=1, \ldots, N\right) .
$$

With probability $\alpha_{j}^{n}$, a packet originating from player $n$ in $N_{j}$ takes a direct path to the center, and otherwise it takes an indirect path: it is first relayed to node $j+1$ and then forwarded to the destination through link $D(k+1)$.

Loss probabilities We consider two types of losses: (1) i.i.d. losses at the relay: a packet originating from node $k$ is lost if relayed to node $k+1$ with probability $q_{k}$. (2) collision losses on the links $D_{k}$ : whenever an arrival occurs while there is another packet in service then there is a loss. The transmission duration of a packet in link $D(k)$ is exponentially distributed with parameter $\mu_{k}$.

The total flow sent to the link $D(k)$ consists of the superposition of (1) the Poisson flows that arrive at node $k$ and are transmitted over $D(k)$, and (2) the Poisson flow originated in node $k-1$ consisting of the packets that were not lost in the relay to node $k$.

Thus the total rate of arrivals to $D(k)$ is

$$
R(k)=\sum_{n=1}^{N} \phi_{k}^{n} \alpha_{k}^{n}+\phi_{k-1}^{n}\left(1-\alpha_{k-1}^{n}\right)\left(1-q_{k}\right)
$$


The loss probability of packets at $D(k)$ is

$$
P(k)=\frac{R(k)}{R(k)+\mu_{k}}
$$

By the same arguments, the loss probability of packets at $D(k+1)$ is

$$
P(k+1)=\frac{R(k+1)}{R(k+1)+\mu_{k+1}}
$$

The total rate of losses of packets of player $(n, k)$ is

$$
J_{k}^{n}(\alpha)=\phi_{k}^{n}\left(\alpha_{k}^{n} P(k)+\left(1-\alpha_{k}^{n}\right)\left[q_{k}+\left(1-q_{k}\right) P(k+1)\right]\right)
$$

In the rest of the paper we assume that $\phi_{k}^{n}, \mu_{k}$ and $q_{k}$ are constant, independent of $k$ and $n$.

\section{The globally optimal solution and the equilibrium}

\subsection{Minimizing average loss probability}

Consider a symmetric multi-strategy $\alpha$ for all players, i.e. in which $\alpha_{k}^{n}$ are the same for all players $(n, k)$. Without loss of generality, let $\phi=1 / N$. Then the rate of arrival of packets at the links $D(k)$ is $R=1-(1-\alpha) q$. The loss probabaility on link $D(k)$ is

$$
P_{l}=\frac{R}{R+\mu}=1-\frac{\mu}{1-(1-\alpha) q+\mu}
$$

so the global loss probability is

$$
\pi=P_{l}+(1-\alpha) q
$$

It is minimized at $\alpha=1$, which means that all players take direct path to the destination.

\subsection{Equilibrium.}

Assume that player $(n, k)$ deviates and plays $b$ instead of playing $\alpha$. Let $u$ be the multi-strategy after the deviation. Then

$$
\begin{gathered}
R(k, u)=[\alpha+(1-\alpha)(1-q)] \frac{N-1}{N}+[b+(1-\alpha)(1-q)] \frac{1}{N} \\
=\frac{b-\alpha}{N}+(1-\alpha)(1-q) \\
R(k+1, u)=[\alpha+(1-\alpha)(1-q)] \frac{N-1}{N}+[\alpha+(1-b)(1-q)] \frac{1}{N}
\end{gathered}
$$




$$
=\alpha+(1-\alpha)(1-q)-(1-q) \frac{b-\alpha}{N}
$$

The loss probability for a player $(k, n)$ is given by

$$
J_{k}^{n}(u)=\frac{1}{N}(b P(k, u)+(1-b)[q+(1-q) P(k+1, u)])
$$

where the path loss probabilities $P(k, u)$ and $P(k+1, u)$ are given in (1)-(2).

To obtain the equilibrium, we:

1. differentiate $J_{k}^{n}(u)$ with respect to $b$, and obtain (using Maple) the expression in Figure 6. Equating the expression to 0 allows us to obtain the best response action $b=f(a)$ that minimizes the loss probabilities of a player when all others use $\alpha$.

2. obtain the symmetric equilibrium by computing the fixed point of the mapping $b=f(\alpha)$. This leads to

$$
\alpha^{*}=-\frac{N \mu q-N q^{2}+N q+q^{2}-2 q+1}{N q^{2}-q^{2}+2 q-2}
$$

If $0<\alpha^{*} \leq 1$, then it is a symmetric equilibrium. If the fixed point is greater than 1 , then the symmetric equilibrium is the policy $\alpha=1$ for all players. In that case, the equilibrium is globally optimal and only the direct links are used. Thus, the equilibrium coincides with the globally optimal policy for all $q$ large enough.

\subsection{Best response}

As already mentioned, we are able with the help of Maple to get an explicit cumbersome expression for the best response. This allows us to obtain a much simpler expression for the equilibrium (as a function of the parameters). We present in Figure 2 the best response $b$ as a function of $q$.

\subsection{When is the globally optimal policy $\alpha=1$ an equilibrium}

Consider the cost $J_{k}^{n}(b, 1)$ for some player $(n, k)$ where $(b, 1)$ is the policy where all players use $\alpha=1$ and the deviating player $(k, n)$ uses $b$.

Theorem 1. A necessary and sufficient condition for $\alpha=1$ to be a symmetric equilibrium is that

$$
q N>\frac{1}{1+\mu}
$$

Proof. A necessary and sufficient condition for the symmetric policy $\alpha=1$ to be an equilibrium is that the cost for the deviating player be decreasing in $b$ at $b=1$. This is equivalent to the following first-order condition. The derivative 


$$
\begin{aligned}
&\left(-2 a^{2} q^{2}-2 a \mu q^{2}+a q^{3}+4 a^{2} q+2 a \mu q-a q^{2}-2 \mu^{2} q+3 \mu q^{2}-q^{3}-2 a^{2}-a q\right. \\
&+2 \mu^{2}-6 \mu q+3 q^{2} \\
&+\left(-a^{4} q^{6}-a^{3} \mu q^{6}+6 a^{4} q^{5}+3 a^{3} \mu q^{5}+3 a^{3} q^{6}-3 a^{2} \mu^{2} q^{5}+3 a^{2} \mu q^{6}-13 a^{4} q^{4}+4 a\right. \\
&-22 a^{3} \mu q^{3}+51 a^{3} q^{4}-18 a^{2} \mu^{2} q^{3}+13 a^{2} \mu q^{4}+22 a^{2} q^{5}+15 a \mu^{3} q^{3} \\
&-35 a \mu^{2} q^{4}+19 a \mu q^{5}+a q^{6}-\mu^{4} q^{3}+3 \mu^{3} q^{4}-3 \mu^{2} q^{5}+\mu q^{6}-4 a^{4} q^{2} \\
&+24 a^{3} \mu q^{2}-62 a^{3} q^{3}-a^{2} \mu^{2} q^{2}+28 a^{2} \mu q^{3}-64 a^{2} q^{4}-24 a \mu^{3} q^{2}+72 a \mu^{2} q^{3} \\
&-43 a \mu q^{4}-8 a q^{5}+5 \mu^{4} q^{2}-18 \mu^{3} q^{3}+21 \mu^{2} q^{4}-8 \mu q^{5}-8 a^{3} \mu q+36 a^{3} q^{2} \\
&+12 a^{2} \mu^{2} q-62 a^{2} \mu q^{2}+94 a^{2} q^{3}+12 a \mu^{3} q-59 a \mu^{2} q^{2}+37 a \mu q^{3}+26 a q^{4} \\
&-8 \mu^{4} q+39 \mu^{3} q^{2}-57 \mu^{2} q^{3}+26 \mu q^{4}-8 a^{3} q-4 a^{2} \mu^{2}+40 a^{2} \mu q-73 a^{2} q^{2} \\
&+12 a \mu^{2} q+2 a \mu q^{2}-44 a q^{3}+4 \mu^{4}-36 \mu^{3} q+75 \mu^{2} q^{2}-44 \mu q^{3}-8 a^{2} \mu \\
&+28 a^{2} q+4 a \mu^{2}-20 a \mu q+41 a q^{2}+12 \mu^{3}-48 \mu^{2} q+41 \mu q^{2}-4 a^{2}+8 a \mu \\
&\left.\left.-20 a q+12 \mu^{2}-20 \mu q+4 a+4 \mu\right)^{12}+a+3 \mu-3 q+1\right) /\left(a q^{3}-3 a q^{2}\right. \\
&\left.+\mu q^{2}-q^{3}+4 a q-\mu q+3 q^{2}-2 a-3 q+1\right)
\end{aligned}
$$

Fig. 2. Expression for the best response function $b=f(\alpha)$

of the cost of the deviating player evaluated at $b=1$ when all other players use $\alpha=1$ is non-negative. Calculation in Maple yields

$$
\left.\frac{d J_{k}^{n}(b, 1)}{d b}\right|_{b=1}=-\frac{\mu(N \mu q+N q-1)}{(1+\mu)^{2}}
$$

This concludes the proof.

We conclude that if one invests in improving a communication channel thus decreasing the loss probabilities (the parameter $q$ in our case), then as a result we may end up worsening the performance for all the users in the system.

\section{Numerical examples}

With the help of Maple, we obtained a simple expression for the equilibrium as a function of the parameters of the system. The following experiments allow us to get insight on the equilibrium behavior.

\subsection{The equilibrium}

We depict in Figure 3 the parameter $\alpha^{*}$ defining the symmetric equilibrium as a function of the loss rate parameter $q$. The following parameters are fixed: $N=1$ and $\mu=1$. As long as $\alpha^{*}$ is in the unit interval, it is the equilibrium. This is the case for $q \leq 0.5$. For larger $q$, the corresponding symmetric equilibrium is $\alpha=1$. 


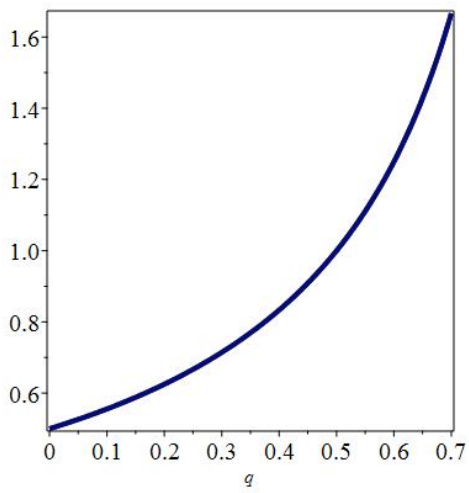

Fig. 3. The symmetric equilibrium as a function of the loss probability parameter $q$.

\subsection{The best response}

In Figure 4, we depict the loss probability of a player that plays $b$ when the others play $\alpha . b$ is varied while $\alpha$ is held fixed. The figure thus allows us to identify the best response $b$. In the case that the best response $b(\alpha)$ to a given $\alpha$ in the unit interval satisfies $b(\alpha)=\alpha$, then $\alpha$ is a symmetric equilibrium.

The following parameters are fixed: $N=1$ and $\mu=1$. There are three curves: the first corresponds to $\alpha=0.5012$ and $q=0.01$, the second to $q=0.3$ and $\alpha=0.55$ and the third to $q=0.9$ and $\alpha=1$. In the two first curves, $b$ for which the derivative of the cost is zero is within the unit interval and is thus the best response to $\alpha$. In the first curve, $\alpha=0.5012$ is a fixed point of the best response function $b(\alpha)$. This confirms that $\alpha=0.5012$ is an equilibrium, which can be seen from Figure 3 .

In the third curve, $b$ that minimizes the cost is larger than 1 and the best response is obtained on the boundary $b=1$ and not on the $b$ for which the derivative w.r.t. $b$ is zero.

\subsection{Non-equilibrium of the globally optimal policy $\alpha=1$}

We depict the best response to the globally optimal policy in Figure 5 , for $N=1$ and $\mu=1$ being held fixed, as a function of the loss parameter $q$. For the above parameters, the best response function is given in Figure 2.

We observe that for all $q<0.5$, the best response to other players sending their traffic through the direct path (i.e. $\alpha=1$ ) is to play $b<1$. We conclude that for these $q, \alpha=1$ is not an equilibrium. In contrast, for all $q \geq 0.5$, the best response to $\alpha=1$ is also $\alpha=1$ and hence the global optimal policy is an equilibrium policy. 


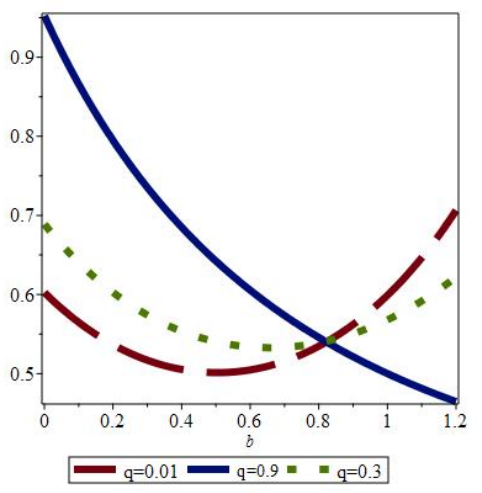

Fig. 4. The symmetric equillibrium as a function of the loss probability parameter $q$.

\subsection{The derivative of the cost of a player}

In order to provide an expression for the equilibrium, we had to differentiate the cost of the player that uses $b$ while others use $a$. Below is long expression obtained by Maple for the derivative w.r.t. $b$ of the cost for the player, i.e. of the loss probability of packets of player $(\mathrm{k}, \mathrm{n})$ who plays $b$ when all others play $\alpha$.

\section{Conclusions}

We have seen that for any choice of system parameters, the equilibrium performance improves when increasing the loss probability parameter $q$ on the relay links. The equilibrium loss probability of a player is thus decreasing in $q$. This is a Braess-type paradox.

Moreover, for any parameters of the system, if the number $N$ of players at each node is large enough, then the globally optimal policy is an equilibrium; this means that in the regime of a large number of players (called a Wardrop equilibrium), the above type of paradox does not occur.

The original Braess paradox [6] was shown to hold in a framework of a very large number of players (Wardrop equilibrium). Later on it was shown to occur also in the case of any number $N>1$ players in [11]. The paradox we introduced, known as the Kameda paradox, does not occur in the case of a very large number of players. This was shown for standard delay-type cost functions in [10] for a triangular network topology.

\section{References}

1. Eitan Altman, Alejandra Estanislao, Manoj Panda. Routing Games on a Circle. NetGCOOP 2011: International conference on NETwork Games, COntrol and OPtimization, Telecom SudParis et Université Paris Descartes, Oct 2011, Paris, France. hal-00644364 


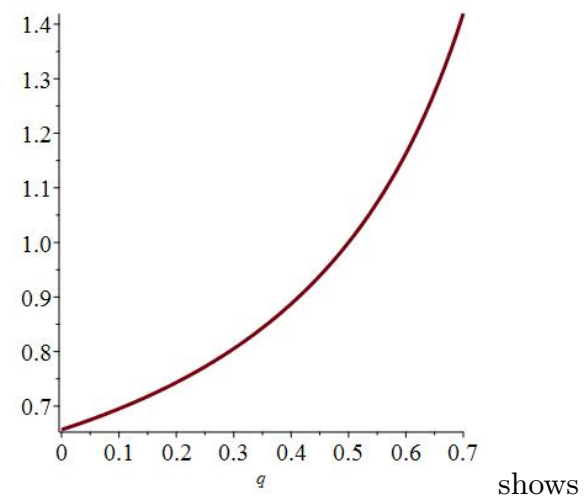

Fig. 5. Sending all the traffic through direct paths is not an equilibrium. The Figure shows the best response of a player when the others use $\alpha=1$ as a function of the loss parameter $q$.

2. Eitan Altman, Joy Kuri, Rachid El-Azouzi. A routing game in networks with lossy links. 7th International Conference on NETwork Games COntrol and OPtimization (NETGCOOP 2014), Oct 2014, Trento, Italy. hal-01066453

3. Eitan Altman, Rachid El-Azouzi, Vyacheslav Abramov. Non-Cooperative Routing in Loss Networks. RR-4405, INRIA. 2002. <inria-00072183> Performance Evaluation Volume 49, Issues 1-4, September 2002, Pages 257-272

4. Eitan Altman, Zhen Liu. Improving the stability characteristics of asynchronous traffic in FDDI token ring. [Research Report] RR-1934, INRIA. 1993, pp.21.

5. Amina Boukoftane, Eitan Altman, Majed Haddad, Nadia Oukid: Paradoxes in a Multi-criteria Routing Game. GAMENETS 2017: 165-172.

6. D. Braess, Uber ein Paradox aus der Verkehrsplanung. Unternehmensforschung 12, $258-268$ (1968)

7. Ramya Burra, Chandramani Singh, Joy Kuri, Eitan Altman. Routing on a Ring Network. Song, Ju Bin; Li, Husheng; Coupechoux, Marceau. Game Theory for Networking Applications, Springer International Publishing, pp.25-36, 2019, 978-3-31993057-2. 10.1007/978-3-319-93058-9³. hal02417

8. Chen X., Doerr B., Hu X., Ma W., van Stee R., Winzen C. (2012) The Price of Anarchy for Selfish Ring Routing Is Two. In: Goldberg P.W. (eds) Internet and Network Economics. WINE 2012. Lecture Notes in Computer Science, vol 7695. Springer, Berlin, Heidelberg

9. M. Haddad, E. Altman and J. Gaillard, "Sequential routing game on the line: Transmit or relay?," 2012 International Conference on Communications and Information Technology (ICCIT), Hammamet, 2012, pp. 297-301.

10. Kameda, H., E. Altman, T. Kozawa and Y. Hosokawa (2000). "Braess-like paradoxes in distributed computer systems," IEEE Trans. Automatic Control, Vol. 45, 1687-1691.

11. Y.A. Korilis, A.A. Lazar, and A. Orda, Avoiding the Braess paradox in noncooperative networks, J. Appl. Prob. 36, 211 - 222 (1999). 


$$
\begin{aligned}
d J b a N & :=\frac{(a+(1-a)(1-q))\left(1-\frac{1}{N}\right)+\frac{b+(1-a)(1-q)}{N}}{(a+(1-a)(1-q))\left(1-\frac{1}{N}\right)+\frac{b+(1-a)(1-q)}{N}+\mu} \\
& +\frac{b}{N\left((a+(1-a)(1-q))\left(1-\frac{1}{N}\right)+\frac{b+(1-a)(1-q)}{N}+\mu\right)} \\
& -\frac{b\left((a+(1-a)(1-q))\left(1-\frac{1}{N}\right)+\frac{b+(1-a)(1-q)}{N}\right)}{\left((a+(1-a)(1-q))\left(1-\frac{1}{N}\right)+\frac{b+(1-a)(1-q)}{N}+\mu\right)^{2} N}-q \\
- & \frac{(1-q)\left((a+(1-a)(1-q))\left(1-\frac{1}{N}\right)+\frac{a+(1-b)(1-q)}{N}\right)}{(a+(1-a)(1-q))\left(1-\frac{1}{N}\right)+\frac{a+(1-b)(1-q)}{N}+\mu}+(1 \\
- & b)\left(\frac{(1-q)(-1+q)}{N\left((a+(1-a)(1-q))\left(1-\frac{1}{N}\right)+\frac{a+(1-b)(1-q)}{N}+\mu\right)}\right. \\
- & \left.\frac{(1-q)\left((a+(1-a)(1-q))\left(1-\frac{1}{N}\right)+\frac{a+(1-b)(1-q)}{N}\right)(-1+q)}{\left((a+(1-a)(1-q))\left(1-\frac{1}{N}\right)+\frac{a+(1-b)(1-q)}{N}+\mu\right)^{2} N}\right)
\end{aligned}
$$

Fig. 6. Expression for the derivative of the cost of the player $(\mathrm{k}, \mathrm{n})$ with respect to $b$.

12. A. Orda, R. Rom and N. Shimkin: Competitive routing in multiuser communication networks. IEEE/ACM Transactions on Networking, vol. 1, no. 5, pp. 510-521, Oct. 1993.

13. Patriksson M. The traffic assignment problem: models and methods. The Netherlands: VSPBV; 1994.

14. J. B. Rosen: Existence and Uniqueness of Equilibrium Points for Concave N-Person Games. Econometrica Vol. 33, No. 3 (Jul., 1965), pp. 520-534 